\section{Monitoring system for infant movement, vocalization, and nurse interaction*}

\author{
LOUIS SIEGEL $†$ \\ Departments of Radiation Biology and Biophysics and Psychology \\ and \\ ARNOLD SAMEROFF \\ Departments of Pediatrics and Psychology \\ University of Rochester School of Medicine and Dentistry \\ Rochester, New York 14620
}

An instrument which monitors and records certain behaviors of the neonate is described. By means of an air-inflated mattress and pressure transducer, the instrument measures whole-body movement. By means of a crib-located microphone, the device measures vocalizations. In addition, the presence and activity of nurse or observer are also monitored and recorded as part of the complete record. Except for the mattress and transducers, the monitor is completely self-contained, even including in its $81 / 2$-in.-high $\times 19$-in.-wide size a 10-channel event recorder.

Overt behavior of the neonate consists, to a large extent, of whole-body movement and vocalization. Of those variables that influence these measurements and that do not arise from the subject, nurse, or other observer, presence and interaction are the most obvious and probable. The instrument to be described ${ }^{1}$ monitors whole-body movement, vocalization, and observer presence and interaction, and provides an event record of these variables as follows: (1) integral of movement per unit time; (2) summated vocalization per unit time; (3) presence of observer; (4) presence of infant in crib; and (5) six different observer or nurse actions. In addition, the amplified raw signals of movement and vocalization are made available for tape-recording, strip chart writeout, or computer processing.

\section{DESCRIPTION}

The system may be considered as three channels: (1) movement channel, (2) vocalization channel, and (3) interaction channel. All three channels are incorporated into a single $83 / 4-i n$. rack-mounted unit and all have outputs in the form of an event mark on the unit's self-contained 10-channel

*This research was supported in part by USPHS Grants $\mathrm{MH}-16544$ and $\mathrm{MH}-11752$, and in part by a contract between the University of Rochester and the Atomic Energy Commission ( $R$ eport No. UR 49-1423). The authors wish to acknowledge the helpful assistance of Mr. Harry Bakow, graduate student in psychology, in setting up and using these monitors. The monitor was designed and constructed with the assistance of Dalyn Data Systems, 94 Castlebar Road, Rochester. New York 14610. The price of the commercial monitor marketed by this company is $\$ 2,500$. event recorder. These channels will be described separately.

\section{Movement Channel}

The input to this channel (Fig. 1) arises from an air-inflated foam-filled crib mattress connected to a Statham pressure transducer by means of a patent air hose of low distensibility. Distortion of the mattress geometry produces an intramattress volume change with a corresponding, but inversely related, pressure change. Differential pressures are on the order of $\pm 0.15 \mathrm{lb} / \mathrm{sq} \mathrm{in}$. Such a range is sensitive to movements produced by respiration alone.

The output of the pressure transducer is amplified by an operational type of instrumentation amplifier, which then drives a sample/hold amplifier operated in a switched-integrator mode. The pressure waveform is therefore integrated over time until rest, whereupon the integration is resumed again starting from 0 . Reset occurs whenever the integrator output

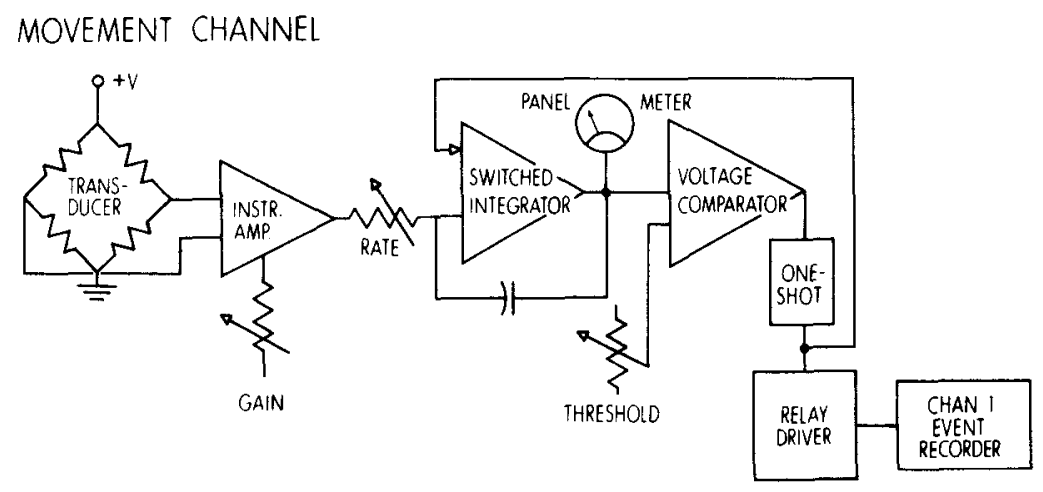

Fig. 1. This channel quantizes whole-body movement by integration. The rate of integration (RATE control) and the upper limit of the integral (THRESHOLD control) are adjustable parameters which are set by using the panel meter as a guide. console is connected to the counter at reaches a preset threshold and is determined by an integrated-circuit comparator. The comparator output resets the integrator and simultaneously delivers a pulse to Channel 1 of the system event recorder. The gain of the instrumentation amplifier, the rate at which the comparator threshold is reached, and the value of the threshold level are adjustable parameters on the monitor console. These adjustments are facilitated by a voltmeter on the console connected to the integrator output, which serves as a visual monitor of momentary movement.

\section{Vocalization Channel}

The input to this channel (Fig. 2) is a high-impedance dynamic microphone mounted in the infant crib. Infant vocalizations are detected by the microphone and delivered to a discrete-component modular-type audio amplifier equipped with sensitivity and frequency controls. The audio output signal is rectified and filtered and then gated with a pulse generated from the $60-\mathrm{Hz}$ line frequency. The output of the gate therefore is a $60-\mathrm{Hz}$ pulse stream for the duration of periods of vocalizations. This pulse stream is delivered to a hybrid type of digital counter whose effective length is presettable for counts of Modulo 120 or 900. The effect of this is such that the counter overflows whenever a total of 2 or $15 \mathrm{sec}$ of vocalization have accumulated. Whenever the counter overflows, a pulse is delivered to Channel 2 of the system event recorder. An indicator lamp on the a point that causes the lamp to flash at a $1 / 10$-sec rate during periods of vocalizations.

\section{Interaction Channel}

Included in this channel are provisions for monitoring (1) presence of infant in crib, (2) presence of 


\section{AUDIO CHANNEL}

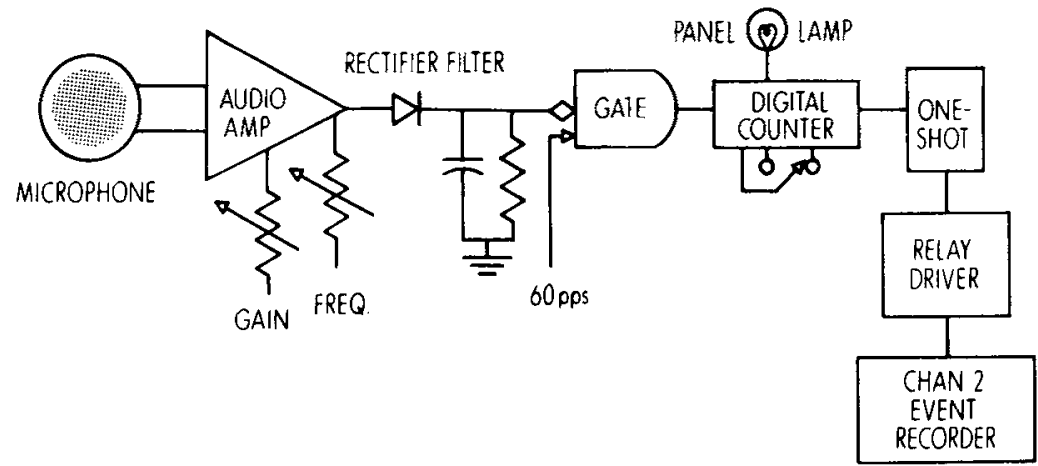

Fig. 2. This channel quantizes vocalization by summing. The counter sums 2 or 15-sec vocalization periods, the size of the quantum being determined by the switch associated with the digital counter. The panel lamp indicates counter activity and serves as a guide for the gain and frequency settings of the audio amplifier.

Table 1

Parts List Instrumentation Amplifier

Switched Integrator

Panel Meter

Voltage Comparator

One Shot

Relay Driver

Event Recorder

Microphone

Audio Amplifier

And Gate

Digital Counter

One Shot

Relay Driver

Event Recorder

Crib Switch

Floor Mat Switch
Pressure Transducer

Figure 1

Statham PM5 \pm 0.15

Burr Brown 3264/14

Burr Brown 4013/25

Simpson 523,0-10VDC

Burr Brown 4032/12C

Digital Equipment Corp. K323

Digital Equipment Corp. K683

Simpson 2755-22201, 24VDC

Figure 2

Shure R5

Amperex PCA-4-18A

Digital Equipment Corp. K113

Digital Equipment Corp. K211

as above

as above

as above

Crib Related

Approved Rubber Co.

141 Lincoln Street

Winthrop, Mass. 02152

Honeywell Microswitch 2PB7

Tapeswitch Corp. CVP1723

observer, and (3) six specific observer-infant interactions. Presence of infant is monitored by a mechanical arrangement sensitive to infant weight. A switch, whose actuation force is slightly more than the tare weight of the crib, mattress, and other paraphernalia usually accompanying the infant is mounted between crib and frame. The addition of the infant to the other crib contents actuates the switch which is connected directly to Channel 3 of the event recorder.

Presence of an observer is defined as an observer directly in front of the crib. This fact is monitored by a floormat switch located at the base of the crib stand and connected directly to Channel 4 of the event recorder.
Specific nurse interaction is recorded by the remaining six channels of the event recorder. Whenever the presence of an observer is detected, a console lamp captioned "NURSE MAKE SELECTION" is lighted. The lamp can be turned off only by making a response on one of six console-mounted pushbuttons captioned with the specific action. These pushbuttons are connected directly to the remaining six event recorder channels.

In addition to the items already mentioned, the monitor console contains four connectors for the attachment of the mentioned channel input devices, as well as for input-amplifier outputs to data processing and storage devices. Parts lists for Figs. 1 and 2 and for crib-related items are presented in Table 1.

\section{APPLICATION}

The monitoring system is utilized for studying newborn activity in general and the endogenous behavioral rhythms of human infants in particular. At birth, infants typically function on a 3- to 4-h schedule, running from one feeding to the next. Sometime during the first few months after birth, infants alter their rhythms to include a circadian component based on the 24-h adult cycle. The most obvious sign of the alteration in rhythm is the elimination of the nighttime (2:00 a.m.) feeding.

The monitoring system is sensitive to the rhythm of infant functioning and provides a complete record of the activity cycles from birth until discharge from the hospital nursery. An advantage of the described monitor is that it requires no electrodes to be placed on the subject, as is typically done elsewhere to record EEG, EKG, or respiratory activity.

A major use for the monitor is in the normative investigation of the development and stability of behavior cycles in the newborn. A second major use is in the description of individual differences in the cycling of infants. These individual differences may arise from (1) differences in genetic history among infants, (2) effects of fetal distress of problems of pregnancy, or (3) actual illness of the child.

Several neurological impairments of the infant are known to produce hyper- or hypoactive behavior in the infant. The monitor provides a continuous activity record, which would permit detection of both extremes of activity while at the same time showing a continuous record of the distribution of activity. Seizure activity could be easily differentiated from a more generalized state of arousal.

\section{REFERENCE}

SANDER, L. W.. \& JULIA, H. Continuous interaction monitoring in the neonate. Psychosomatic Medicine, 1966, 28, 822-835.

\section{NOTE}

1. This instrument is based on a prototype arrangement described in an unpublished manuscript, Continuous interaction monitoring in early infancy: Progress report, by L. W. Sander, G. Stechler, and H. Julia, 1967. 